\title{
Fully Biologically Active In Vitro Transcripts of the Eriophyid Mite-Transmitted Wheat Streak Mosaic Tritimovirus
}

\author{
Il-Ryong Choi, Roy French, Gary L. Hein, and Drake C. Stenger
}

First author: School of Biological Sciences, University of Nebraska, Lincoln 68583; second and fourth authors: USDA-ARS and Department of Plant Pathology, University of Nebraska, Lincoln 68583; and third author: Department of Entomology, University of Nebraska Panhandle Research and Extension Center, Scottsbluff 69361.

Accepted for publication 26 August 1999.

\begin{abstract}
Choi, I.-R., French, R., Hein, G. L., and Stenger, D. C. 1999. Fully biologically active in vitro transcripts of the eriophyid mite-transmitted wheat streak mosaic tritimovirus. Phytopathology 89:1182-1185.

Infectious RNA of wheat streak mosaic virus (WSMV) has been produced using a full-length cDNA clone as a template for in vitro transcription with SP6 RNA polymerase. Infectivity was dependent on the use of template plasmid DNA that had not undergone spontaneous rearrangement during amplification in Escherichia coli. The presence of WSMV in systemically infected wheat plants inoculated with in vitro transcripts was confirmed by reverse-transcription polymerase chain reaction of the WSMV P3 gene and by accumulation of WSMV coat protein as detected by immunoblotting. Maintenance of the full-length WSMV cDNA in the

high copy number plasmid pUC18 was problematic because of spontaneous rearrangement of WSMV sequences during growth in liquid media for more than $\sim 8 \mathrm{~h}$ or if the clone was subcultured. Stability of the WSMV cDNA clone was improved by the use of the low copy number plasmid pACYC177, and it could be grown in large scale volumes (up to 1 liter) of liquid culture for $\sim 14 \mathrm{~h}$ without noticeable rearrangements. Both the original WSMV culture and the progeny virus derived from infectious in vitro transcripts were efficiently transmitted by the natural eriophyid mite vector Aceria tosichella. This is the first report of infectious in vitro transcripts for any eriophyid mite-transmitted plant virus and represents the only monopartite member of the family Potyviridae infecting monocotyledonous hosts for which infectious in vitro transcripts are available.
\end{abstract}

Use of infectious transcripts from full-length clones facilitates manipulation of RNA virus genomes via their cDNA clones. Of the numerous and economically important plant viruses of the family Potyviridae, infectious clones have been reported only for seven monopartite, aphid-transmitted species of the genus Potyvirus that infect dicotyledonous hosts $(3-8,10-12,14)$ and for barley mild mosaic virus (BaMMV) (13). BaMMV is a bipartite, fungaltransmitted species of the genus Bymovirus that infects monocotyledonous hosts. Stability in Escherichia coli has been a common obstacle to the construction and maintenance of full-length cDNA clones to species of the family Potyviridae. In the studies listed above, clone stability has been achieved by the introduction of intron sequences (11), the use of partial clones that are ligated in vitro and subsequently amplified by polymerase chain reaction (PCR) for use as a template for transcription (5), or the growth of the infectious clone in small culture volumes for limited periods (3).

Wheat streak mosaic virus (WSMV) is a monopartite member of the family Potyviridae that is transmitted by the eriophyid mite Aceria tosichella Keifer (1). WSMV is widespread throughout the Great Plains of North America and causes significant losses in wheat (Triticum aestivum L.) (1). The genome organization of the 9,384-nucleotide (nt) RNA of WSMV is similar to those of other monopartite members of the family Potyviridae, although there is

Corresponding author: D. C. Stenger; E-mail address: dstenger@unlnotes.unl.edu

Mention of proprietary or brand names are necessary to report factually on available data; however, the USDA neither guarantees nor warrants the standard of the product, and the use of the name by USDA implies no approval to the exclusion of others that also may be suitable.

Publication no. P-1999-1015-01R

This article is in the public domain and not copyrightable. It may be freely reprinted with customary crediting of the source. The American Phytopathological Society, 1999. substantial sequence divergence relative to other members of the family (15). Phylogenetic analyses (15) indicate that WSMV, along with brome streak mosaic virus (9), are sufficiently different from other members of the family Potyviridae to be placed in the newly recognized genus Tritimovirus. Previously, there were no infectious clones available for any eriophyid mite-transmitted plant virus. Thus, the infectious cDNA clone of WSMV described here represents the first such reagent for an eriophyid mite-transmitted virus and represents the first infectious clone of a monopartite member of the family Potyviridae capable of infecting monocotyledonous plants. We also describe the use of a low copy number plasmid as a simple means of improving clone stability in E. coli.

\section{MATERIALS AND METHODS}

Construction of a full-length cDNA clone of WSMV. Overlapping partial cDNA clones of the WSMV Sidney 81 isolate have been described (15) and were assembled here to construct a fulllength WSMV cDNA (Fig. 1). The following nt sequences of WSMV (GenBank accession no. AF057533) were found in the respective clones: 1 to 330 (p5'-4), 185 to 5,038 (pN511-16), 4,870 to 8,704 (pN511-36), 5,437 to 9,384 plus six $3^{\prime}$-terminal adenosine (A) residues (pT52-16), and 8,920 to 9,384 plus $1053^{\prime}$-terminal A residues (pN522-10). A DNA fragment containing the fusion of the SP6 promoter and the WSMV 5' terminus was generated by PCR using $P f u$ polymerase with $5^{\prime}-4$ as a template. The plus-sense primer (5'-GTGTGTCGACCGATTTAGGTGACACTATAGAAATTAAACCAACCCAAATCG-3') contained a SalI site (bold) and the SP6 promoter (underlined) immediately upstream of the WSMV 5' terminus (the A residue adjacent to the 3 -proximal $\mathrm{G}$ residue of the SP6 promoter). The minus-strand primer (5'-GTTGGTCGACGCTTCCTGGTCCGTGGTG-3') also contained a SalI site (bold). The resulting PCR product was digested with SalI and cloned into pUC18 to obtain pUCSP6-5. A 4.8-kilobase pair (kbp) fragment 
was recovered from pN511-16 after complete digestion with SalI (cleaving a polylinker site $3^{\prime}$ proximal to the WSMV cDNA insert) and partial digestion with BssHII (WSMV nt coordinate 209). The resulting fragment was ligated into pUCSP6-5 (after removal of a small DNA fragment from pUCSP6-5 delimited by the BssHII and 3'-proximal SalI sites) to produce pUCSP6-5/N511-16. The 3 -terminal sequences of pT52-16 were replaced with those of pN522-10 by ligating a 3.9-kbp fragment of pT52-16, generated by digestion with HpaI (WSMV nt coordinate 9,301) and NotI (cleaving a 5'-proximal polylinker site), with pN522-10 (after removal of a small DNA fragment from pN522-10 delimited by the same HpaI and 5'-proximal NotI polylinker sites) to produce pT52-16/N522-10. The cDNA fragment of pT52-16/N522-10 was digested with AatII (a 3'-proximal polylinker site), made bluntended by removal of $3^{\prime}$ overhangs with T4 DNA polymerase, and then digested with Eco47III (WSMV nt coordinate 7,984) to isolate a $1.5-\mathrm{kbp}$ fragment. Subsequently, the $1.5-\mathrm{kbp}$ fragment was inserted into pUCSP6-5/N511-16 to replace the DNA fragment between the Eco47III site (WSMV nt coordinate 5,001) and the 3'proximal SmaI polylinker site, giving pSP6WSMV $\Delta$ E47. Finally, pSP6WSMV was constructed by inserting an Eco47III fragment (WSMV nt coordinates 5,001 to 7,984) derived from pN511-36 into the unique Eco47III site in pSP6WSMV $\Delta$ E47. The resultant plasmid, pSP6WSMV, is a high copy number, pUC18-based construct that has an SP6 promoter followed by the full-length cDNA sequence of WSMV and an additional 105 3'-terminal A residues followed by unique NotI, SpeI, and SacII sites (Fig. 1). The $5^{\prime}$ end of the RNA produced by SP6 transcription of pSP6WSMV is predicted to initiate at a guanosine $(\mathrm{G})$ residue, resulting in an in vitro transcript that contains a 5'-proximal $G$ residue that does not occur in authentic WSMV RNA. To produce a low copy number version of the full-length WSMV cDNA clone, the entire SP6 promoter-WSMV cDNA insert was excised from pSP6WSMV by digestion with SalI and SpeI and cloned into the XhoI and NheI sites of the low copy number plasmid vector pACYC177 (New England Biolabs, Inc., Beverly, MA) to produce pACYC-WSMV.
In vitro transcription and infectivity assays. Plasmids used as templates (12.5 to $25 \mu \mathrm{g}$ per reaction) for in vitro transcription were linearized with SacII (pSP6WSMV) or NotI (pACYC-WSMV). In vitro transcription was performed in the presence or absence of $2 \mathrm{mM}$ cap analogue $\left(\mathrm{m}^{7} \mathrm{G}\left[5^{\prime}\right] \mathrm{ppp}\left[5^{\prime}\right] \mathrm{G}\right)$ using the SP6 MAXIscript kit (Ambion, Austin, TX) as recommended by the manufacturer. Quantity and quality of RNA transcripts were assessed on $1 \%$ agarose gels. An equal volume of $2 \%$ sodium pyrophosphate containing $2 \mathrm{mg}$ of bentonite per $\mathrm{ml}$ was added to the in vitro transcription products, and the mixture was inoculated onto Carborundum-dusted leaves of 8- to 10-day-old wheat plants (cv. Centurk). Each plant was inoculated with 0.5 to $1 \mu \mathrm{g}$ of transcript. Plants inoculated with in vitro transcripts were visually evaluated for WSMV infection. Infection was verified by reverse-transcription (RT) PCR of the WSMV P3 gene using primers specific for the P3 gene of WSMV (5'-CCGGATGGGAATGGCAACAGCGAATTGT-3' and 5'-CCGGATCCTAATAAGTTGTGATAAAGCC-3'). Independent verification of infection was accomplished by immunoblotting total soluble protein extracts with WSMV coat protein (CP) antibodies as described (2).

Eriophyid mite transmission assays. Virus-free colonies of the eriophyid mite $A$. tosichella were maintained on caged wheat plants (cv. Arapahoe). Wheat plants (cv. Centurk) infected with the authentic WSMV Sidney 81 isolate or with progeny virus derived from in vitro transcripts served as source plants in vector transmission assays. Virus-free A. tosichella (20 per plant) were placed on WSMV-infected source plants or on noninfected wheat plants for a 7-day acquisition access period (AAP). Following the AAP, groups of five A. tosichella were moved to wheat test plants (cv. Arapahoe) for a 96-h inoculation access period (IAP). Following the IAP, all test plants were treated with carbofuran to eliminate mites. To prevent movement of the mites, all source and test plants were caged throughout the study. Test plants were evaluated for WSMV infection 21 days postinoculation by enzyme-linked immunosorbent assay (ELISA) using antibodies to WSMV CP.
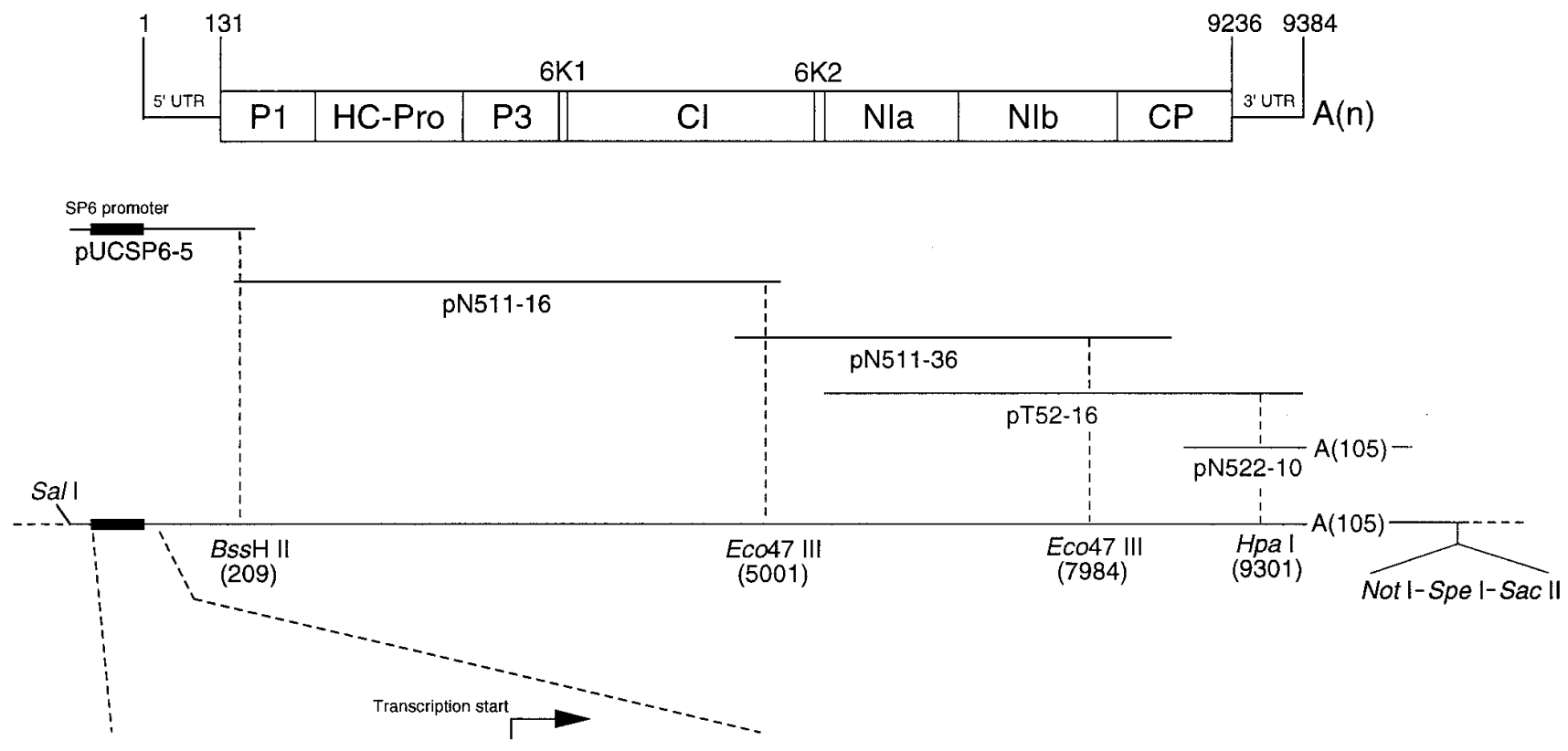

ATTTAGGTGACACTATAGAAATTAAACC

SP6 Promoter

Fig. 1. Construction of the full-length cDNA clone (pSP6WSMV) of wheat streak mosaic virus (WSMV). The genome organization of WSMV is depicted on the top. The cDNA portions of pUCSP6-5, pN511-16, pN511-36, pT52-16, and pN522-10, used for the construction of pSP6WSMV, are aligned according to their size and relative locations in the WSMV genome. Restriction endonuclease sites and nucleotide positions used for ligation of adjacent cDNA fragments are indicated below the schematic diagram of pSP6WSMV. The junction of the SP6 promoter (underlined) and the 5' end of the WSMV cDNA produced by polymerase chain reaction is detailed on the bottom. The arrow indicates the predicted transcriptional start (G residue) in the SP6 promoter. 


\section{RESULTS AND DISCUSSION}

Stability of full-length WSMV cDNA clones. We often noticed spontaneous rearrangement of intermediate constructs, as well as of the full-length WSMV cDNA, during amplification in the high copy number plasmid pUC18. Similar construct instability has been noted with cDNA clones of potyviral genomes $(5,10,11)$. In particular, pSP6WSMV was highly unstable when amplified in $E$. coli strains DH5 $\alpha$ and XL2-Blue. Low yields of nonrearranged pSP6WSMV from these two E. coli strains could be recovered by limiting growth periods to $>6 \mathrm{~h}$ in small-scale $(2 \mathrm{ml})$ culture vol-

TABLE 1. Infectivity of in vitro transcripts of wheat streak mosaic virus (WSMV) on wheat

\begin{tabular}{lrcccc}
\hline Inoculum & Exp. 1 & Exp. 2 & Exp. 3 & Total \\
\hline Mock & $0 / 10^{\mathrm{b}}$ & $0 / 8$ & $0 / 7$ & $0 / 25(0 \%)$ \\
WSMV Sidney 81 & $20 / 20$ & $\mathrm{ND}^{\mathrm{d}}$ & $\mathrm{ND}$ & $20 / 20(100 \%)$ \\
pSP6WSMV (uncapped) & $0 / 20$ & $\mathrm{ND}$ & $\mathrm{ND}$ & $0 / 20(0 \%)$ \\
pSP6WSMV (capped) & $13 / 21$ & $28 / 41$ & $19 / 28$ & $60 / 90(67 \%)$ \\
pACYC-WSMV (uncapped) & $15 / 19$ & $11 / 17$ & $15 / 16$ & $41 / 52(78 \%)$ \\
pACYC-WSMV (capped) & $8 / 14$ & $21 / 29$ & $18 / 32$ & $47 / 75(63 \%)$ \\
pACYC-WSMV (capped, subcultured) & $5 / 25$ & $20 / 26$ & $\mathrm{ND}$ & $25 / 51(49 \%)$ \\
\hline
\end{tabular}

a Transcript template recovered from JM109 cells after retransformation.

${ }^{b}$ Number of plants inoculated / number of plants infected.

${ }^{c}$ Sap from wheat plants infected with authentic WSMV.

${ }^{\mathrm{d}} \mathrm{ND}=$ not determined.
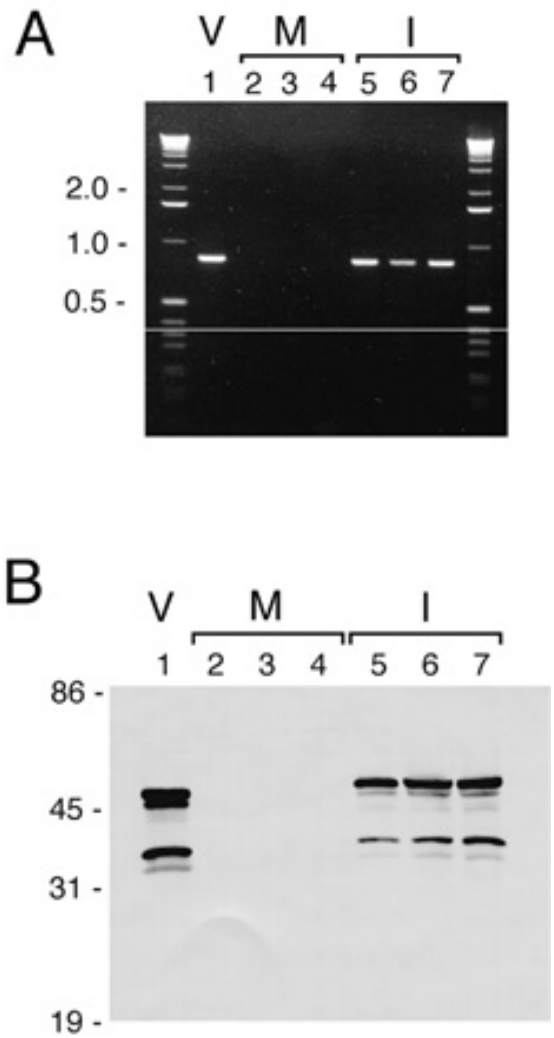

Fig. 2. Detection of a virus-specific gene or protein from plants inoculated with in vitro transcripts from pSP6WSMV. A, Detection of the wheat streak mosaic virus (WSMV) P3 gene sequence by reverse-transcription polymerase chain reaction. Samples were prepared from wheat plants inoculated with the authentic WSMV Sidney 81 isolate $(\mathrm{V}$, lane 1), mock-inoculated with buffer alone (M, lanes 2 to 4), or inoculated with in vitro transcripts using pSP6WSMV as a template (I, lanes 5 to 7). A 1-kbp ladder is shown on both sides of the gel, and the position and sizes (kbp) of DNA fragments are indicated on the left. B, Immunoblot analysis of WSMV coat protein. Total soluble protein samples were prepared from the same plants as described in $\mathbf{A}$, separated by polyacrylamide electrophoresis, and analyzed by immunoblotting with antibodies raised to the coat protein of WSMV. The position and size (kDa) of protein standards are indicated on the left. umes. Longer growth periods, larger culture volumes, or subculture of the clone in DH5 $\alpha$ or XL2-Blue consistently resulted in extensive rearrangements of the WSMV cDNA sequence as determined by alteration of endonuclease restriction profiles. The use of the E. coli strain JM109 as a host resulted in a higher yield of plasmid when grown in 200-ml culture volumes for $\sim 8 \mathrm{~h}$. However, most plasmid preparations grown under these conditions contained a proportion of rearranged molecules and, upon subculture of pSP6WSMV in JM109, only rearranged plasmids were recovered. As an example, endonuclease restriction mapping of pSP6WSMV recovered after subculture indicated a $1-\mathrm{kb}$ insertion in the $\mathrm{P} 3$ gene between nts 2,779 and 3,111 (data not shown). Nonrearranged pSP6WSMV could be propagated by retransformation of JM109 with the plasmid, although it was necessary to rescreen transformants by restriction mapping to select a nonrearranged clone.

The full-length WSMV cDNA cloned into the low copy number plasmid pACYC-177 (pACYC-WSMV) was sufficiently stable to permit amplification for 12 to $14 \mathrm{~h}$ in culture volumes up to 1 liter without noticeable rearrangement. However, to remove residual chromosomal DNA from plasmids extracted from large-scale cultures, it was necessary to isolate supercoiled plasmid DNA on $\mathrm{CsCl}$ gradients, a practice common in the early 1980s but largely abandoned since the introduction of high copy number, pUC-based plasmids. Furthermore, subculture of pACYC-WSMV did not result in plasmid preparations that had undergone obvious rearrangement, as determined by endonuclease restriction mapping.

In vitro transcripts of WSMV cDNA are infectious. Twothirds of the wheat plants inoculated with capped in vitro transcripts from pSP6WSMV developed mosaic and stunting symptoms typical of infection by WSMV (Table 1). Capped transcripts synthesized from pACYC-WSMV also were infectious, with infectivity averaging $63 \%$ over three experiments (Table 1). Symptoms could be seen as early as 6 days after inoculation with in vitro transcripts, and there were no apparent differences between symptoms produced by authentic WSMV or those produced by RNA transcribed from either the high or low copy number plasmid constructs. These results indicate that the presence of one nonviral $\mathrm{G}$ residue at the $5^{\prime}$ end of the RNA did not destroy infectivity. Although a single experiment with uncapped transcripts derived from pSP6WSMV did not result in infection, uncapped transcripts derived from pACYC-WSMV were consistently infectious in three independent experiments (Table 1) and represent the first demonstra-

TABLE 2. Aceria tosichella transmission of the wheat streak mosaic virus (WSMV) progeny virus derived from infectious in vitro transcripts

\begin{tabular}{lcc}
\hline Inoculum & Source plant & Test plant \\
\hline WSMV Sidney 81 & A & $2 / 2^{\mathrm{a}}$ \\
& $\mathrm{B}$ & $2 / 3$ \\
& $\mathrm{C}$ & $2 / 2$ \\
& $\mathrm{D}$ & $2 / 2$ \\
& $\mathrm{E}$ & $2 / 2$ \\
Total & $\mathrm{F}$ & $4 / 4$ \\
pSP6WSMV progeny & All & $14 / 15(93 \%)$ \\
& $\mathrm{A}$ & $1 / 2$ \\
& $\mathrm{~B}$ & $1 / 1$ \\
& $\mathrm{C}$ & $3 / 3$ \\
& $\mathrm{D}$ & $3 / 3$ \\
Total & $\mathrm{E}$ & $2 / 3$ \\
Virus-free & $\mathrm{F}$ & $3 / 3$ \\
& $\mathrm{All}$ & $13 / 15(87 \%)$ \\
& $\mathrm{A}$ & $0 / 1$ \\
Total & $\mathrm{B}$ & $0 / 4$ \\
& $\mathrm{C}$ & $0 / 3$ \\
& $\mathrm{D}$ & $0 / 4$ \\
& $\mathrm{E}$ & $0 / 4$ \\
& $\mathrm{~F}$ & $0 / 3$ \\
& $\mathrm{All}$ & $0 / 19(0 \%)$ \\
& & \\
& & \\
& &
\end{tabular}

${ }^{a}$ Number of plants infected / number of plants inoculated. 
tion of cap-independent infectivity with a cDNA clone of the family Potyviridae.

In vitro transcription using plasmid templates recovered from JM109 after retransformation produced infectious RNA transcripts (Table 1, experiments 2 and 3), indicating that pSP6WSMV and pACYC-WSMV could be maintained and propagated by retransformation of JM109, although it was necessary to verify that retransformants of pSP6WSMV had not spontaneously rearranged. Subculture of pACYC-WSMV did not result in gross rearrangement as did pSP6WSMV, and transcripts produced from subcultured pACYC-WSMV were infectious (Table 1). Because rearranged plasmid was always recovered after subculture of pSP6WSMV, infectivity of its transcripts was not tested.

To verify infection of wheat by the in vitro transcripts, WSMV P3 gene sequences were amplified by RT-PCR. As shown in Figure 2A, an 850-bp RT-PCR product corresponding to the P3 gene sequence was detected from a plant inoculated with the WSMV Sidney 81 isolate and from plants inoculated with in vitro transcripts. As expected, no P3 RT-PCR product was amplified from mock-inoculated plants (Fig. 2A). Accumulation of WSMV CP was observed in the plant inoculated with the authentic WSMV Sidney 81 isolate, as well as in those plants inoculated with in vitro transcripts (Fig. 2B). The multiple banding pattern observed for WSMV $\mathrm{CP}$ has been noted and attributed to partial degradation of $\mathrm{CP}$ as the infection progresses (2).

Progeny virus of cloned WSMV is transmissible by $A$. tosichella. Both the WSMV Sidney 81 isolate and the progeny virus derived from cloned WSMV transcripts were transmissible by $A$. tosichella (Table 2). All groups of mites given an AAP on source plants infected with the WSMV Sidney 81 isolate or with the progeny virus derived from in vitro transcripts were able to transmit virus. Transmission efficiencies ranged between 50 to $100 \%$ for groups of five A. tosichella transferred to test plants for a 96-h IAP (Table 2). All symptomatic test plants were confirmed to be infected with WSMV by ELISA, while all nonsymptomatic test plants exposed to viruliferous $A$. tosichella and test plants exposed to virus-free $A$. tosichella were negative for WSMV infection in ELISA tests (data not shown).

Progeny virus derived from WSMV cDNA is fully biologically active. The WSMV Sidney 81 isolate can systemically infect the corn (Zea mays L.) inbred line SDP2, whereas the WSMV type isolate cannot (R. French, unpublished data). Sap from symptomatic wheat infected with the progeny virus derived from in vitro transcripts was inoculated to SDP2 seedlings. Inoculated SDP2 seedlings (four of four) developed mosaic symptoms identical to that induced by the Sidney 81 isolate, indicating that the cloned WSMV cDNA retained this biological property.

As the progeny virus derived from in vitro transcripts was efficiently transmitted by A. tosichella, the full-length WSMV cDNA construct provides the critical reagent needed to apply reverse genetics for elucidating viral determinants of eriophyid mite transmission and host range. Also, the availability of an infectious cDNA clone of the WSMV genome will facilitate characterization of gene function in this economically important plant virus and will permit direct comparison of gene function between eriophyid mite- and aphid-transmitted members of the family Potyviridae.

\section{ACKNOWLEDGMENTS}

This work was supported, in part, by funding from the University of Nebraska Center for Biotechnology to I.-R. Choi. We thank J. S. Hall and S. Steele for excellent technical assistance; and R. Donis, K. Scheets, T. J. Morris, and L. Lane for helpful comments.

\section{LITERATURE CITED}

1. Brakke, M. K. 1971. Wheat streak mosaic virus. CMI/AAB Descriptions of Plant Viruses No. 48. Association of Applied Biologists, Wellesbourne, United Kingdom.

2. Brakke, M. K., Skopp, R. N., and Lane, L. C. 1990. Degradation of wheat streak mosaic virus capsid protein during leaf senescence. Phytopathology 80:1401-1405.

3. Dolja, V. V., McBride, H. J., and Carrington, J. C. 1992. Tagging of plant potyvirus replication and movement by insertion of $\beta$-glucuronidase into the viral polyprotein. Proc. Natl. Acad. Sci. U.S.A. 89:10208-10212.

4. Domier, L. L., Franklin, K. M., Hunt, A. G., Rhoads, R. E., and Shaw, J. G. 1989. Infectious in vitro transcripts from cloned cDNA of a potyvirus, tobacco vein mottling virus. Proc. Natl. Acad. Sci. U.S.A. 86:3509-3513.

5. Fakhfakh, H., Vilaine, F., Makni, M., and Robaglia, C. 1996. Cell-free cloning and biolistic inoculation of an infectious cDNA of potato virus Y. J. Gen. Virol. 77:519-523.

6. Flasinski, S., Gunasinghe, U. B., Gonzales, R. A., and Cassidy, B. G. 1996. The cDNA sequence and infectious transcripts of peanut stripe virus. Gene 171:299-300.

7. Gal-on, A., Antignus, Y., Rosner, A., and Raccah, B. 1991. Infectious in vitro RNA transcripts derived from cloned cDNA of the cucurbit potyvirus, zucchini yellow mosaic virus. J. Gen. Virol. 72:2639-2643.

8. Gal-on, A., Meri, E., Huet, H., Hua, W. J., Raccah, B., and Gaba, V. 1995. Particle bombardment drastically increases the infectivity of cloned DNA of zucchini yellow mosaic potyvirus. J. Gen. Virol. 76:3223-3227.

9. Gotz, R., and Maiss, E. 1995. The complete nucleotide sequence and genome organization of the mite-transmitted brome streak mosaic rymovirus in comparison with those of potyviruses. J. Gen. Virol. 76:20352042.

10. Jakab, G., Doz, E., Brigneti, G., Baulcombe, D., and Malone, P. 1997. Infectious in vitro transcripts from a full-length cDNA clone of PVY-N605, a Swiss necrotic isolate of potato virus Y. J. Gen. Virol. 78:3141-3145.

11. Johansen, I. E. 1996. Intron insertion facilitates amplification of cloned virus cDNA in Escherichia coli while biologically activity is re-established after transcription in vivo. Proc. Natl. Acad. Sci. U.S.A. 93:12400-12405.

12. Maiss, E., Timpe, U., Brisske-Rode, A., Lesemann, D. E., and Casper, R. 1992. Infectious in vivo transcripts of a plum pox potyvirus full-length cDNA clone containing the cauliflower mosaic virus $35 \mathrm{~S}$ promoter. J. Gen. Virol. 73:709-713.

13. Meyer, M., and Dessens, J. T. 1997. 35 S promoter-driven cDNAs of barley mild mosaic virus RNA1 and RNA2 are infectious on barley plants. J. Gen. Virol. 78:3147-3151.

14. Riecmann, J. L., Lain, S., and Garcia, J. A. 1990. Infectious in vitro transcripts from a plum pox potyvirus cDNA clone. Virology 177:710-716.

15. Stenger, D. C., Hall, J. S., Choi, I.-R., and French, R. 1998. Phylogenetic relationships within the family Potyviridae: Wheat streak mosaic virus and brome streak mosaic virus are not members of the genus Rymovirus. Phytopathology 88:782-787. 